\title{
Objective Meaning of Logical Knowledge
}

\author{
Vitaly Ivlev \\ Bauman Moscow State Technical University \\ 5/1, 2nd Baumanskaya St., Moscow 105005 Russia \\ E-mail: vitalijivlev@yandex.ru
}

\author{
Yuriy Ivlev \\ Lomonosov Moscow State University \\ Leninskie gory 1, Moscow 119991 Russia \\ E-mail: ivlev.logic@yandex.ru
}

\begin{abstract}
Logic, same as other sufficiently developed sciences, features two types of knowledge: empirical and theoretical. At the empirical level a distinction is drawn between objects described by logical and non-logical terms. It is possible to divide the first group further, into those that describe objective reality and those that describe cognition. The second group consists of those designating or denoting objects, their properties and relations, including functional ones. The main feature of theoretical knowledge is that it deals with models of the objects studied. A model is an object that is similar to the original object, but simplified, and serves the purpose of cognition. Systems of modal logic are built upon models of empirical objects.
\end{abstract}

Keywords-empirical knowledge; theoretical knowledge; logical terms; theoretical terms; model; logical system

\section{INTRODUCTION}

Aleksandr Aleksandrovich Zinoviev distinguishes two types of knowledge in logic, social studies and other sciences: empirical and theoretical [1] [2] [3]. This article attempts to consider logical knowledge from this standpoint, building a range of logical systems using the semantic method and formalising these systems. We understand a logical system to be a set of relations between statements according to logical forms.

\section{EMPIRICAL KNOWLEDGE IN LOGIC}

Logic studies relations between thoughts according to socalled logical forms. The logical form of a thought is its structure, which is revealed by means of partial abstraction from the meanings and implications of non-logical terms.

In order to educe the logical form of a thought it is necessary to know the types of non-logical terms, which entails a necessity to know the types of objects designated (or denoted) by these terms. This leads to logic distinguishing semantic categories of language expressions, that is, classes of expressions organised on the basis of their meanings. The main objects at the empirical level of cognition are objects, their properties and relations, including functional ones. The following non-logical terms correspond to these objects: proper names, common names, property symbols, relation symbols, object function symbols.

Partial abstraction from the meanings and implications of non-logical terms means that the information on term type is retained along with the information signifying where the terms were the same and where they were different.

The other part of logical knowledge that is denoted by the logical form of a thought is the information represented by logical terms. There exists a certain objective basis for classifying terms into logical and non-logical, which is the fact that non-logical terms are as a rule specific to each area of knowledge (each science), while logical terms are not, again as a rule. However, this is not enough to classify terms into logical and non-logical. We have to admit that ultimately, this classification is based on agreement. For example, deontic terms such as "permissible", "forbidden", "obligatory" may be seen not as logical terms but rather those of ethics or law. This results in difficulties defining the subject matter of logic. According to Yuriy V. Ivlev, logic is a science dealing with thought forms and relations between thoughts according to logical forms. If there exists no clear criterion for classifying terms into logical and non-logical, it affects the definition of the subject matter of logic. On the other hand, this situation occurs in many sciences. There exist difficulties defining the subject matter of psychology, mathematics and so on. Apparently chemistry is free from this difficulty.

What information on reality (both objective and subjective) do logical terms convey at the empirical level of cognition? They may be divided into those that describe objective reality and those that describe cognition. The first group consists of terms that are denoted by the following words and word combinations, for example: "is/are", "if... then", "and", "or", "either ... or", "necessary (in fact)", "possible (in fact)", "obligatory (by law or, in a certain sense, objectively)", "permissible (in the same sense)"; the second contains quantitative (quantifier) words "consequently ("if ... then")", "compatible (in terms of truth or falsity)", "necessary (in terms of logic)", "possible (in terms of logic)".

Of course, these words and word combinations are not logical terms yet. These words and word combinations denote various terms in natural language. For example, natural language uses the "if ... then" conjunction in different meanings, in other words, it denotes different logical terms. Let us compare the following sentences: "if the position of the Moon changes, tides happen" and "if all students are learners, then some learners are students". In the first case this conjunction means that an event physically depends on another, and in the second case it denotes the relation of logical consequence between statements. There also exist 
other meanings of this conjunction. This leads to different propositions featuring the grammatical conjunction "if ... then" being distinguished.

Conditional.A conditional proposition states that one situation causes another.

A proposition is a conditional one if its antecedent describes a situation that is a sufficient condition for the sitation described by the consequent. The conditional "if ... then" conjunction is indicated by an arrow (). In logical notation: AB.

Counterfactual.The counterfactual "if ... then" conjunction is indicated the following way: A proposition with this conjunction means that the situation described by its antecedent does not exist, but if it did, the situation described by the consequent would exist. For example, if Petrov were a president, he would not be living in a communal apartment. The counterfactual conjunction is widely used in studies of history, including counterfactual historical simulation [4] [5] [6]. In logical notation: AB.

Profactual. Example: "If I am a student, then I must take exams". The antecedent is a proposition that is certainly true. The antecedent then represents a sufficient condition for the situation described by the consequent. The profactual "if ... then" conjunction is indicated as follows: . In logical notation: AB.

Limiting conditional. Here is an example. "If a dog bites off a chicken's left leg, the chicken will be able to stand" (on its remaining leg). The antecedent here represents a condition this is insufficient for the situation described by the consequent not to exist. We can rephrase the proposition as follows: If a dog only bites off a chicken's left leg, the chicken will be able to stand. A limiting conditional relation is represented by the symbol. In logical notation: $\mathrm{AB}$.

A.A. Zinoviev stated the problem of developing logical branches for every science. He managed to solve this problem for some of them. He developed logical physics and logical social studies, the former explicitly and the latter implicitly. What does the "logical branch of a science" phrase mean? It means creating a specification of logical terms that this branch of science uses for reasoning. For example, it may be possible to differentiate between types of conditional propositions (propositions of the $\mathrm{AB}$ form), that is, a cause-and-effect relation between situations A and B, where situation A denotes a condition which causes the situation B if added to existing conditions, and so on. This logical branch is also responsible for distinguishing types of non-logical terms used in the science, since this is required for detecting logical forms and establishing relations between thoughts according to those.

We only considered several of the range of meanings expressed by the "if ... then" conjunction used at the empirical level of cognition. Various meanings of a number of other logical connectors can be found in [7]. It should be noted that we are discussing not the meanings that people use in the process of communication but those logical concepts that these conjunctions may denote.
Here is an example of detecting meanings for the concepts of "necessary", "possible" and "accidental" at the empirical level of cognition in order to design respective logical operators.

Accidentality and necessity, possibility and actuality may be employed in systems and models of counterfactual historical simulation, scientific methodology, scientific cognition and so on [8] [9]. Vitaliy Yu. Ivlev distinguishes several cases of employing modal concepts in biology [10] [11] [12].

Case 1. Changes in population as a result of genetic drift The following concepts are in use here. Accident 1: combinations of various alleles in a gamete are random. Accident 2: individuals select procreation partners randomly. Accident 3: variations in gene pool (genetic drift) may happen randomly in small isolated populations. We can combine accidents 1 and 2: an event is random if no external or internal factors determine whether it exists or not. Let us name it accident $(1,2)$.

The theory of large-scale stochastic phenomena may aid in explaining and describing the accident 3. In logic and sociology, a statistical population is a large-scale stochastic phenomenon (a set of all the events constituting a phenomenon). In this case, it will be a population that has not lost part of its individuals yet. A data sample is a set of objects selected for investigation. Here it will be the part of the original population that has split off and may develop into a new population.

These are the principles behind drawing data samples:

1) objects should be selected from all the subsets of the statistical population; in this case we should select individuals from all those subsets that differ in individual genotypes; since we are dealing with natural selection here, the split-off part must contain representatives of all genotypes for genetic drift not to occur;

2) the number of objects sampled from the statistical population subsets should be proportional to the subset volumes; for example, if there are three genotype-based subsets, one of which contains $1 / 2$ of all individuals and the other two contain 1/4 each, half the sampling set should also contain representatives of the first genotype, and the second half should comprise equal numbers of individuals with the two remaining genotypes; if nature "does not conform" to this, genetic drift may occur;

3) the number of objects selected for the investigation must be optimum. What happens in a situation known as genetic drift? Nature itself "breaks the rules" outlined above or at least one of those. This is a way of saying, of course, that in fact the part that splits off is too small, or it does not contain representatives of every genotype, or the representative ratio is different from that of the genotype ratio in the original population. As a result, the gene pool reproduction conditions get violated due to effects that are external as regards the original population. Therefore, the accident 3 implies a violation of the gene pool reproduction conditions due to external factors (that is, changes in the population's existence conditions); in general,accident 3 is 
something that happens due to some external effect upon the object of cognition (an individual, a physical object, a system and so on).

Genetic drift is a stochastic phenomenon. If the conditions and principles outlined above are observed, there is no genetic drift, that is, the law of population gene pool stability over several generations, known as the HardyWeinberg principle, is in force. This is true for a large population where no mutations happen and Mendel's second law, the Law of Independent Assortment, applies. There is no point then speaking about adhering to the methodology principles presented above, save for the principle based on the law of averages, since the proposition does not concern the sample but the whole statistical population. The HardyWeinberg principle is in force for the case when a number of individuals split off from the original population as well, supposing all the conditions and principles noted above are satisfied. It posits the necessity of population gene pool stability. How could we characterise this concept of necessity? Can we say that this concept of necessity is a concept that depends on the essence of the system? The answer is yes. Our phenomenon is gene pool stability over several generations. The essence (in this regard) is something that determines this stability, the conditions and principles outlined above, which in this case should be certainly regarded as system properties.

Therefore, the accident (accident 3) issomething determined by the external existence conditions of a system, and the necessity (necessity 1 ) is something determined by the essence of a system. We can consider these categories to be conjugate. Accident as equiprobability, as randomness of an event or its absence is the accident $(1,2)$.

Case 2. Organism traits necessary for or accidental to its survival. We mean the population when we say "organism", since it is the population that evolves. The system with regard to which we solve the problem of necessity or accident consists of the population and its habitat. Habitat conditions are the essence of our system. A trait necessary for organism survival is one whose preservation (but not appearance) is determined by the essence of the system. This is necessity not due to origin (necessity 2). A trait is impossible not due to origin if the system (that is, the population and its habitat) determines the death of organisms possessing this trait. A trait is accidental not due to origin if the system does not determine either its preservation or loss.

Case 3. Mutations. The following concepts of necessity and accidentality are in use here. Mutations caused artificially, through deliberately affecting chromosomes and genes, are conditionally necessary; in other words,conditional necessityis a phenomenon the existence or appearance of which is determined by external circumstances. Conditional accident: mutations happen as a result of natural causes, but in a minority of individuals, not in all of them, and this dependence is not deterministic. Spontaneous accidentmeans mutations happening occasionally for no obvious reasons, only in specific individuals (one should note that it were biologists who introduced the term "spontaneous").
Case 4. Genetic dependence of organism traits. The following concepts of necessity, accidentality and possibility are in use when studying this trait dependence. A trait being unambiguously determined by the genetic code of an organism is a necessity. A trait being ambiguously determined by genetic material specifics is an accident. Traits ambiguously determined by genetic anomalies are possible. Generally possibility can be represented by a number between 0 and 1 .

Based on this, we can state several generalised concepts of necessity and accidentality.

The first concept of necessity:something (a property, ratio, relation, event and so on) is necessary if it is unambiguously determined by the internal factors of a thing, system and so on, or their external existence conditions. Let us use examples to illustrate the concept of being unambiguously determined. For instance, conductivity in metals is unambiguously determined by the presence of free electrons, while some diseases are ambiguously determined by genetic or chromosomal anomalies, meaning that these anomalies may or may not lead to these diseases manifesting, depending on certain circumstances. Ambiguous determination exists objectively. That is, in the case of unambiguous determination a respective cause is a sufficient condition for a certain effect to emerge. In the case of ambiguous determination (quasidetermination) a cause is a sufficient condition for one of several specific effects, but it is impossible in principle to find out which effect it would be. The following concept of accidentality is conjugate to this concept of necessity: something (a property, ratio, relation, event and so on) is accidental if it is determined, but only ambiguously, by the internal factors of a thing (a system and so on), or its external existence conditions.

The second concept of necessity: a necessity is unambiguously determined by the essence of a thing, system and so on. An example of essence is the genetic code of an organism. Accident is something that is ambiguously determined by the essence of a thing (a system and so on), and also that which is determined (unambiguously or ambiguously) by external or inconsequential internal factors.

The third concept is the concept of functional necessity: a trait is necessary if the existence condition for its carrier unambiguously determines the presence of this trait as essential to the carrier's existence, for example, for survival of an organism. A trait is accidental if its absence does not preclude the existence of its carrier. Accidentality not due to origin is an example of this type of accidentality.

The fourth concept is conditional necessity: a phenomenon is necessary if its existence or appearance is unambiguously determined by external circumstances. Mutations caused artificially, through deliberately affecting chromosomes and genes, are an example of this type of necessity. This concept is also applicable in the case of social events. Accident is something which is ambiguously determined by the essence of a thing, system and so on.

A statement is true, that is, its value is $t$, if the situation it describes occurs in reality (taking temporal characteristics 
into account). Then, a situation that occurs in reality may be necessary or accidental in one of the meanings outlined above; for example, a situation described by a statement is unambiguously determined by the essence of a thing or system (the second concept of necessity presented above), or is not unambiguously determined. It is unfeasible to rate the statement as necessary in the latter case. There are two ways out of this situation. The first involves introducing situation assessment: "occurs in reality", "factually (physically) necessary" ("unambiguously determined by the essence of a thing"). The second involves introducing a respective assessment of the statement describing the necessary situation along with the concept of truth. The first way out is unacceptable for us since it contradicts established tradition. In the second case we cannot find a suitable expression to assess our statement. We had to rate not only situations but statements as necessary or accidental. Let us assume these expression assessments in the latter case to have a meaning different from that of situation assessments.

We define the first two conjugate concepts of necessity and accidentality based on unambiguous and ambiguous situation determinations. Suppose the situation described by the statement A occurs in reality and its presence is unambiguously determined (by external or internal conditions of a thing, system and so on), that is, the statement $\mathrm{A}$ has a value of $\mathrm{t}^{\mathrm{n}}$ (true and necessary, or necessary truth). It is evident that the statement " $\mathrm{A}$ is necessary" is true, that is, the determination stated occurs in reality. Then there is a question whether this unambiguous determination is itself unambiguously determined by external or internal conditions, that is, whether we assign the value $\mathrm{t}^{\mathrm{n}}$ or $\mathrm{t}^{\mathrm{c}}$ (true and accidental, accidental truth) to the statement A for the case of assigning the value to the statement A mentioned above. We will limit ourselves to discussing the determination of conditions that unambiguously or ambiguously determine the situation. Let our situation, for example, a disease manifesting in an organism, be unambiguously determined by anomalies internal to the organism. The latter in turn are unambiguously determined by other anomalies in the organism, and so on. In this case, we should assign the value $\mathrm{t}^{\mathrm{n}}$ to the statement $\mathrm{A}$. If $\mathrm{A}$ has the value of $\mathrm{t}^{\mathrm{c}}$, that is, the situation described by the statement A occurs in reality and is not unambiguously determined by internal factors, for example, then these factors may in turn be ambiguously determined by other internal factors and so on. д. In this case, we should assign the value $\mathrm{f}^{\mathrm{c}}$ (false and accidental) to both statements $\mathrm{A}$ and $\mathrm{A}$. Likewise we obtain the value $\mathrm{f}^{\mathrm{i}}$ (false and necessary, necessary falsity, meaning the absence of a situation is unambiguously defined).

By summarising the modal terms isolated above, we arrive at the following definitions for alethic modal operators in "Table I".

TABLE I. DEFINITIONS For ALETHIC MODAL OPERATORS

\begin{tabular}{|c|c|c|c|c|c|c|c|c|c|}
\hline & $\mathbf{a}$ & b & c & d & e & f & g & h & i \\
\hline A & A A & A A & A A & A & A A & A A & $\mathrm{A} A$ & A A & A A \\
\hline $\mathrm{t}^{\mathrm{n}}$ & $\mathrm{t} \quad \mathrm{t}$ & $\mathrm{t}^{\mathrm{n}} \quad \mathrm{t}^{\mathrm{t}}$ & $t^{n} \quad t^{n}$ & $\mathrm{t}^{\mathrm{c}} \quad \mathrm{t}$ & $\mathrm{t}^{\mathrm{c}} \quad \mathrm{t}^{\mathrm{c}}$ & $t \quad t$ & $\mathrm{t} \mathrm{t}$ & $t^{n} \quad t^{n}$ & $\mathrm{t}^{\mathrm{c}} \quad \mathrm{t}^{\mathrm{c}}$ \\
\hline $\mathrm{t}^{\mathrm{c}}$ & $f \quad t$ & $\mathrm{f}^{\mathrm{c}} \quad \mathrm{t}^{\mathrm{c}}$ & $\mathrm{f}^{\mathrm{i}} \mathrm{t}^{\mathrm{n}}$ & $\mathrm{f}^{\mathrm{c}}$ & $f^{i} t^{n}$ & $\mathrm{f}^{\mathrm{i}} \mathrm{t}^{\mathrm{n}}$ & $\mathrm{f}^{\mathrm{c}} \quad \mathrm{t}^{\mathrm{c}}$ & f $\quad t$ & \\
\hline $\mathrm{f}^{\mathrm{i}}$ & f $\mathrm{f}$ & f $\mathrm{f}$ & $f^{i} f^{i}$ & $\mathrm{f}^{\mathrm{c}}$ & $f^{f^{i}} f^{c}$ & $\mathrm{f} \mathrm{f}$ & f f & $f^{i} f^{i}$ & $f^{c} f^{c}$ \\
\hline $\mathrm{f}^{\mathrm{c}}$ & $\mathrm{f} \mathrm{t}$ & $\mathrm{f}^{\mathrm{c}} \mathrm{t}^{\mathrm{t}}$ & $\mathrm{f}^{\mathrm{i}} \mathrm{t}^{\mathrm{n}}$ & $f^{c}$ & $f^{\prime} t^{n}$ & $f^{\prime} t^{n}$ & $\mathrm{f}^{\mathrm{c}} \mathrm{t}^{\mathrm{c}}$ & $\mathrm{f} \mathrm{t}$ & $\mathrm{f} \mathrm{t}$ \\
\hline
\end{tabular}

$\mathrm{f}$ and $\mathrm{t}$ respectively mean "either false and impossible, or false and accidental", "either true and necessary, or true and accidental".

Logical systems, meaning sets of logical laws using the modal operators and empirical connectors outlined above (conditional relation, conjunctive relations: simultaneous conjunction $\left(\&^{=}\right)$, sequential conjunction $\left(\&^{\mathrm{n}}\right.$, where $\left.\mathrm{n} 1\right)$ and others) have not yet been developed.

One conclusion drawn from the facts stated above may be that one of the components of logical knowledge (possibly even the main component) is the knowledge of objective reality, containing the knowledge dealing with the types of objects of cognition, knowledge of relations between situations and knowledge of how situations may determine other situations [13]. This knowledge comprises the logical ontology [14]. It proves the teaching of A.A. Zinoviev that all sciences, including logic, have an empirical component. In order to create integrated empirical logic we need to classify the laws featuring various logical terms. These are various logical connectors, for example, various logical conjunctions (simultaneous, successive and so on), various connectors that the "if ... then" conjunction denotes in natural languages (various types of conditional relations, limiting conditional relation and so on), various modal terms, for example, various alethic modal terms, deontic operators.

Empirical knowledge also contains knowledge of relations between thoughts according to forms, such as relations of succession, compatibility in terms of truth and falsity and so on. At the empirical level the counterexample method most often serves to establish these relations. The types of knowledge mentioned above comprise the logical anthropology.

\section{THEORETICAL KNOWLEDGE IN LOGIC}

The main feature of theoretical knowledge is that it deals with models of the objects studied. According to A.A. Zinoviev, people invent models of logical objects of cognition as amateurs, and specialist logicians do it as professionals.

A model is an object that is similar to the original object, but simplified, and serves the purpose of cognition. As a rule, it not only simplifies the original but also distorts it. Simplification of cognition compensates for this distortion of reality. In order to see an object better, one sometimes needs 
to move a certain distance away from it. This may lead to detail being invisible.

Material implication () may serve as an example of a theoretical term in logic. This logical term defined through a truth table communicates the general meaning of a conditional relation () and succession relation (). Its definition does not account for certain content specific to the conditional conjunction, namely how the antecedent and the consequent are related by implication (there occurs an abstraction from this relation).

Alethic modal systems are based on the definitions of modal operators presented above and on definitions of other logical terms by means of quasifunctions, including the following in "Table II", "Table III", "Table VI", and "Table V":

TABLE II. ALETHIC MODAL SySTEMS

\begin{tabular}{|l|l|l|l|l|}
\hline \multicolumn{1}{|c|}{$(+) \supset$} & \multicolumn{1}{c|}{$\mathbf{t}^{\mathbf{n}}$} & \multicolumn{1}{c|}{$\mathbf{t}^{\mathrm{c}}$} & \multicolumn{1}{c|}{$\mathbf{f}^{\mathrm{i}}$} & \multicolumn{1}{c|}{$\mathbf{f}^{\mathrm{c}}$} \\
\hline $\mathrm{t}^{\mathrm{n}}$ & $\mathrm{t}^{\mathrm{n}}$ & $\mathrm{t}^{\mathrm{c}}$ & $\mathrm{f}^{\mathrm{i}}$ & $\mathrm{f}^{\mathrm{c}}$ \\
\hline $\mathrm{t}^{\mathrm{c}}$ & $\mathrm{t}^{\mathrm{n}} / \mathrm{t}^{\mathrm{c}}$ & $\mathrm{t}^{\mathrm{c}}$ & $\mathrm{f}^{\mathrm{c}}$ & $\mathrm{f}^{\mathrm{c}}$ \\
\hline $\mathrm{f}^{\mathrm{i}}$ & $\mathrm{t}^{\mathrm{n}}$ & $\mathrm{t}^{\mathrm{n}}$ & $\mathrm{t}^{\mathrm{n}}$ & $\mathrm{t}^{\mathrm{n}}$ \\
\hline $\mathrm{f}^{\mathrm{c}}$ & $\mathrm{t}^{\mathrm{n}} / \mathrm{t}^{\mathrm{c}}$ & $\mathrm{t}^{\mathrm{c}}$ & $\mathrm{t}^{\mathrm{c}}$ & $\mathrm{t}^{\mathrm{n}} / \mathrm{t}^{\mathrm{c}}$ \\
\hline
\end{tabular}

TABLE III. DEFINITIONS OF OTHER LOGICAL TERMS (1)

\begin{tabular}{|l|l|l|l|l|}
\hline$(-) \supset$ & \multicolumn{1}{|c|}{$\mathbf{t}^{\mathrm{n}}$} & \multicolumn{1}{c|}{$\mathbf{t}^{\mathrm{c}}$} & \multicolumn{1}{|c|}{$\mathbf{f}^{\mathrm{i}}$} & \multicolumn{1}{c|}{$\mathbf{f}^{\mathrm{c}}$} \\
\hline $\mathrm{t}^{\mathrm{n}}$ & $\mathrm{t}^{\mathrm{n}}$ & $\mathrm{t}^{\mathrm{c}}$ & $\mathrm{f}^{\mathrm{i}}$ & $\mathrm{f}^{\mathrm{c}}$ \\
\hline $\mathrm{t}^{\mathrm{c}}$ & $\mathrm{t}^{\mathrm{n}} / \mathrm{t}^{\mathrm{c}}$ & $\mathrm{t}^{\mathrm{c}}$ & $\mathrm{f}^{\mathrm{c}}$ & $\mathrm{f}^{\mathrm{c}}$ \\
\hline $\mathrm{f}^{\mathrm{i}}$ & $\mathrm{t}^{\mathrm{n}}$ & $\mathrm{t}^{\mathrm{n}}$ & $\mathrm{t}^{\mathrm{n}}$ & $\mathrm{t}^{\mathrm{n}}$ \\
\hline $\mathrm{f}^{\mathrm{c}}$ & $\mathrm{t}^{\mathrm{n}}$ & $\mathrm{t}^{\mathrm{c}}$ & $\mathrm{t}^{\mathrm{c}}$ & $\mathrm{t}^{\mathrm{n} / \mathrm{t}^{\mathrm{c}}}$ \\
\hline
\end{tabular}

TABLE IV. DEFINITIONS OF OTHER LOGICAL TERMS (2)

\begin{tabular}{|l|l|l|l|l|}
\hline()$\supset$ & $\mathbf{t}^{\mathrm{n}}$ & \multicolumn{1}{c|}{$\mathbf{t}^{\mathrm{c}}$} & \multicolumn{1}{c|}{$\mathbf{f}^{\mathrm{i}}$} & \multicolumn{1}{c|}{$\mathbf{f}^{\mathrm{c}}$} \\
\hline $\mathrm{t}^{\mathrm{n}}$ & $\mathrm{t}^{\mathrm{n}}$ & $\mathrm{t}^{\mathrm{c}}$ & $\mathrm{f}^{1}$ & $\mathrm{f}^{\mathrm{c}}$ \\
\hline $\mathrm{t}^{\mathrm{c}}$ & $\mathrm{t}^{\mathrm{n}}$ & $\mathrm{t}^{\mathrm{c}}$ & $\mathrm{f}^{\mathrm{c}}$ & $\mathrm{f}^{\mathrm{c}}$ \\
\hline $\mathrm{f}^{\mathrm{i}}$ & $\mathrm{t}^{\mathrm{n}}$ & $\mathrm{t}^{\mathrm{n}}$ & $\mathrm{t}^{\mathrm{n}}$ & $\mathrm{t}^{\mathrm{n}}$ \\
\hline $\mathrm{f}^{\mathrm{c}}$ & $\mathrm{t}^{\mathrm{n}}$ & $\mathrm{t}^{\mathrm{c}}$ & $\mathrm{t}^{\mathrm{c}}$ & $\mathrm{t}^{\mathrm{c}}$ \\
\hline
\end{tabular}

TABLE V. DEFINITIONS OF OTHER LOGICAL TERMS (3)

\begin{tabular}{|l|l|}
\hline \multicolumn{1}{|c|}{$\mathbf{A}$} & \multicolumn{2}{c|}{$\mathbf{A}$} \\
\hline $\mathrm{t}^{\mathrm{n}}$ & $\mathrm{f}^{\mathrm{i}}$ \\
\hline $\mathrm{t}^{\mathrm{c}}$ & $\mathrm{f}^{\mathrm{c}}$ \\
\hline $\mathrm{f}^{\mathrm{i}}$ & $\mathrm{t}^{\mathrm{n}}$ \\
\hline $\mathrm{f}^{\mathrm{c}}$ & $\mathrm{t}^{\mathrm{c}}$ \\
\hline
\end{tabular}

Based on the definitions supplied, we can build logical systems

Sa-, Sa, Sa+, Sb-, Sb, Sb+, Sc-, Sc, Sc+, Sd-, Sd, Sd+, $\mathrm{Se}-, \mathrm{Se}, \mathrm{Se}+, \mathrm{Sf}-, \mathrm{Sf}, \mathrm{Sf}+, \mathrm{Sg}-, \mathrm{Sg}, \mathrm{Sg}+, \mathrm{Sh}-, \mathrm{Sh}, \mathrm{Sh}+, \mathrm{Si}-, \mathrm{Si}$, $\mathrm{Si}+$. The lowercase character in the name of a system corresponds to the method of defining modal terms; the symbols + , - or their absence correspond to the method of determining implication. th and tc are the isolated values.

A formalisation of semantically defined systems will be those calculi that share all the axiom schemata of classical predicate calculus, the modus ponens rule, and the following axiom schemata:
$\mathrm{AA} ; \quad \mathrm{AA} ; \quad \mathrm{AA} ; \quad \mathrm{A}(\mathrm{AB}) ; \quad \mathrm{B}(\mathrm{AB}) ; \quad \mathrm{B}(\mathrm{AB}) ; \quad \mathrm{A}(\mathrm{AB}) ;$ $(\mathrm{AB})(\mathrm{AB})$.

We use the letter $\mathrm{S}$ to designate the calculus which is an extension of classical predicate calculus through these eight axiom schemata.No semantics is developed for this calculus, neither is there any informative explanation of the meaning behind the modal logic terms it uses.

Other calculi designated the same as the semantically defined systems are built by means of extending the calculus $\mathrm{S}$ through the following axiom schemata.

$$
\begin{aligned}
& \text { Sa-: }(\mathrm{AB})(\mathrm{AB}) \text {. } \\
& \text { Sa: }(\mathrm{AB})(\mathrm{AB}) ;(\mathrm{AB})(\mathrm{AB}) ;(\mathrm{AB})(\mathrm{A}(\mathrm{B}(\mathrm{AB}))) . \\
& \text { Sa+: }(\mathrm{AB})(\mathrm{AB}) ;(\mathrm{AB})(\mathrm{AB}) . \\
& \text { Sb-: }(\mathrm{AB})(\mathrm{AB}) ; \mathrm{AA} ; \mathrm{AA} ; \mathrm{AA} ; \mathrm{AA} ; \mathrm{AA} ; \mathrm{AA} . \\
& \text { Sb: }(\mathrm{AB})(\mathrm{AB}) ;(\mathrm{AB})(\mathrm{AB}) ;(\mathrm{AB})(\mathrm{A}(\mathrm{B}(\mathrm{AB}))) \mathrm{AA} ; \mathrm{AA} ; \\
& \text { AA; AA; } \mathrm{AA} ; \mathrm{AA} .
\end{aligned}
$$

$\mathrm{Sb}+$ ( $\mathrm{AB})(\mathrm{AB}) ;(\mathrm{AB})(\mathrm{AB}) ; \mathrm{AA} ; \mathrm{AA} ; \mathrm{AA} ; \mathrm{AA} ; \mathrm{AA} ; \mathrm{AA}$.

Calculi Sc-, Sd-, Se-, Sf-, Sg-, Sh-, Si- comprise the $(\mathrm{AB})(\mathrm{AB})$ axiom schema.

Calculi Sc, Sd, Se, Sf, Sg, Sh, Si comprise the (AB)(AB); $(\mathrm{AB})(\mathrm{AB}) ;(\mathrm{AB})(\mathrm{A}(\mathrm{B}(\mathrm{AB})))$ axiom schemata.

Calculi $\mathrm{Sc}+, \mathrm{Sd}+, \mathrm{Se}+, \mathrm{Sf}+, \mathrm{Sg}+, \mathrm{Sh}+, \mathrm{Si}+$ comprise the $(\mathrm{AB})(\mathrm{AB}) ;(\mathrm{AB})(\mathrm{AB})$ axiom schemata.

Those calculi the designation of which contains the same lowercase character, for example, calculi $\mathrm{Sc}, \mathrm{Sc}, \mathrm{Sc}+$, only differ in the $\{(\mathrm{AB})(\mathrm{AB})\}$, $\{(\mathrm{AB})(\mathrm{AB}) ;(\mathrm{AB})(\mathrm{AB}) ;(\mathrm{AB})(\mathrm{A}(\mathrm{B}(\mathrm{AB})))\}, \quad\{(\mathrm{AB})(\mathrm{AB})$; $(\mathrm{AB})(\mathrm{AB})\}$ axiom schemata.

The remaining additional axiom schemata of these calculi are the same. Here are these two final additional axiom schemata.

Calculi Sc-, Sc, Sc+. AA; AA; AA; AA.

Calculi $\mathrm{Sd}-, \mathrm{Sd}, \mathrm{Sd}+. \mathrm{A}^{*}$, where $\mathrm{A}^{*}$ is a modalised formula.

Calculi Se-, Se, Se+. A; A; AA; AA; A(AA); A(AA); A(AA); A(AA).

Calculi Sf-, Sf, Sf+. A(AA); A(AA); A(AA); A(AA).

Calculi Sg-, Sg, Sg+. A(AA); A(AA); A(AA); A(AA).

Calculi Sh-, Sh, Sh+. AA; AA; AA; AA.

Calculi $\mathrm{Si}-, \mathrm{Si}, \mathrm{Si}+$ A; A; AA; AA.

\section{CONCLUSION}

In order to develop a complex logic as envisioned by A.A. Zinoviev, in our opinion, at the first stage of cognition we should integrate the systems of alethic modal logic describing ontological modalities by means of using various symbols to designate different types of these terms, different implications and other logical terms. This also concerns 
other logical terms (logical and deontic modalities and others) [15].

\section{REFERENCES}

[1] Zinoviev A.A. Logical and physical succession. // Problems of logic in scientific cognition. Moscow, 1964, pp. 87-150.

[2] Zinoviev A.A. Complex logic. Moscow, Nauka Publ., 1970.

[3] Zinoviev A.A. The logic of science. Moscow, Mysl Publ., 1971.

[4] Nekhamkin V.A. Counterfactual studies in historical cognition: genesis and methodology. Moscow, MAKS Press, 2006.

[5] Nekhamkin V.A. A counterfactual challenge of the past: Ways of negotiation // Herald of the Russian Academy of Sciences. 2017. Vol 87. Issue 2. P. 191-198. DOI: 10.1134/S1019331617020046.

[6] Nekhamkin V.A. Sources of Alternate History and Its Methodological Significance // Istoriya-electronnyi nauchno-obrazovatelnyi zhurnal. 2017. Vol. 8. Issue 2. DOI 10.18254/S0001696-4-1.

[7] Ivlev Yu.V. Ternary calculi and their interpretations. // Studies in logic and philosophy. No. 4. Moscow, 2010. Pp.57-71.

[8] Lebedev S.A. The Structure of Scientific Rationality // Voprosy filosofii. 2017. № 5. P. 66-79.

[9] Ivlev V.Yu., Oseledchik M.B. Methodological principles for the introduction of modality categories in modern scientific cognition // Proceedings of the 3-rd International Conference on Arts, Design, and Contemporary Education. (ICADCE 2017) Advances in Social Science, Education and Humanities Research». Paris: Atlantis Press, 2017. V.144. Pp.541-545. https://doi.org/10.2991/icadce-17.2017.128

[10] Ivlev V. Yu. "Necessity", "accidentality" and "possibility" in biology and their philosophical generalisation. // Categories. Journal of philosophy. 1997, no. 2.

[11] Ivlev V. Yu. Categories of necessity, accidentality and possibility: their meaning and methodological role in scientific cognition. // Philosophy and society. 1997, no. 3.

[12] Ivlev V.Yu., Lepskaya N.D. Modalities and logic // "Proceedings of the 2017 2nd International Conference on Contemporary Education, Social Sciences and Humanities". (ICCESSH 2017). Part of the series ASSEHR. Moscow, Russia. V.124. P.79-86. http://dx.doi.org/10.2991/iccessh-17.2017.18

[13] Voyshvillo E.K., Degtyarev M. G. Logic and elements of epistemology and scientific methodology. Moscow, Nauka Publ., 1994.

[14] Ivlev Yu.V. Certain problems of "tractatus logico-philosophicus" as seen in contemporary logic // Proc. of the research seminar of the Logical Center of the Institute of Philosophy of the Russian Academy of Sciences. 2000. Vol. 14. Pp. 156-165.

[15] Ivlev Yu.V. Quasi-matrix logic as a paraconsistent logic for dubitable information. // Logic and Logical Philosophy. Volume 8 (2000), pp. 91-97. http://dx.doi.org/10.12775/LLP.2000.006 